\title{
COMPENSATORY PUFFING WITH LOWER NICOTINE \\ CONCENTRATION E-LIQUIDS INCREASES CARBONYL EXPOSURE IN E-CIGARETTE AEROSOLS
}

Kośmider L, $\mathrm{PhD}^{1}$, Kimber $\mathrm{CF}^{2}$, Kurek $\mathrm{J} \mathrm{PhD}^{3}$, Corcoran $\mathrm{O} \mathrm{PhD}^{4}$, Dawkins $\mathrm{LE} \mathrm{PhD}^{5}$

${ }^{1}$ School of Pharmacy with the Division of Laboratory Medicine in Sosnowiec, Medical University of Silesia, Department of General and Analytical Chemistry, Jagiellonska 4 Street 41200 Sosnowiec, Poland

${ }^{2}$ Drugs and Addictive Behaviours Research Group, School of Psychology, University of East London, Water Lane, E15 4LZ, UK

${ }^{3}$ Institute of Occupational Medicine and Environmental Health, Koscielna 13 Street, 41-200 Sosnowiec, Poland

${ }^{4}$ Medicines Research Group, School of Sports, Health and Bioscience, University of East London, Water Lane, E15 4LZ, UK

${ }^{5}$ Division of Psychology, School of Applied Sciences, 103 Borough Road, London South Bank University, London, SE1 0AA, UK

Corresponding author: Catherine F Kimber, MBPsS, Drugs and Addictive Behaviours Research Group, School of Psychology, University of East London, Water Lane, E15 4LZ, UK; Tel: +44 (0) 208223 4592; Email: c.kimber@uel.ac.uk 


\section{COMPENSATORY PUFFING WITH LOWER NICOTINE \\ CONCENTRATION E-LIQUIDS INCREASES CARBONYL EXPOSURE \\ IN E-CIGARETTE AEROSOLS}

Key words: Nicotine, E-cigarette, Electronic Nicotine Delivery Devices, Carbonyls, Puffing

Topography, Tobacco Product Directive

\section{ABSTRACT \\ Introduction}

Article 20 of the Tobacco Products Directive (EU-TPD) specifies that e-liquids should not contain nicotine in excess of $20 \mathrm{mg} / \mathrm{mL}$, thus many vapers may be compelled to switch to lower concentrations and in so doing, may engage in more intensive puffing. This study aimed to establish whether more intensive puffing produces higher levels of carbonyl compounds in ecigarette aerosols.

\section{Methods}

Using the HPLC-UV diode array method, four carbonyl compounds (formaldehyde, acetaldehyde, acetone and acrolein) were measured in liquids and aerosols from nicotine solutions of 24 and $6 \mathrm{mg} / \mathrm{mL}$. Aerosols were generated using a smoking machine configured to replicate puffing topography data previously obtained from 12 experienced e-cigarette users.

\section{Results}

Carbonyl levels in aerosols from the puffing regimen of $6 \mathrm{mg} / \mathrm{mL}$ were significantly higher ( $<<0.05$ using independent samples t-tests) compared with those of $24 \mathrm{mg} / \mathrm{mL}$ nicotine. For the 6 
and $24 \mathrm{mg} / \mathrm{mL}$ nicotine aerosols respectively, means $\pm \mathrm{SD}$ for formaldehyde levels were $3.41 \pm 0.94$, and $1.49 \pm 0.30 \mu \mathrm{g}$ per hour $(\mu \mathrm{g} / \mathrm{h})$ of e-cigarette use. Means \pm SD for acetaldehyde levels were $2.17 \pm 0.36$ and $1.04 \pm 0.13 \mu \mathrm{g} / \mathrm{h}$. Means \pm SD for acetone levels were $0.73 \pm 0.20$ and $0.28 \pm 0.14 \mu \mathrm{g} / \mathrm{h}$. Acrolein was not detected.

\section{Conclusions}

Higher levels of carbonyls associated with more intensive puffing suggest that vapers switching to lower nicotine concentrations (either due to the EU-TPD implementation or personal choice), may increase their exposure to these compounds. Based on real human puffing topography data, this study suggests that limiting nicotine concentrations to $20 \mathrm{mg} / \mathrm{mL}$ may not result in the desired harm minimalization effect.

\section{IMPLICATIONS}

More intensive puffing regimens associated with the use of low nicotine concentration e-liquids can lead to higher levels of carbonyl generation in the aerosol. Although in need of replication in a larger sample outside a laboratory, this study provides pragmatic empirical data on the potential risks of compensatory puffing behaviours in vapers, and can help to inform future regulatory decisions on nicotine e-liquid concentrations. The cap on nicotine concentration at 20 $\mathrm{mg} / \mathrm{mL}$ set by the EU-TPD may therefore have the unintended consequence of encouraging use of lower nicotine concentration e-liquid in turn, increasing exposure to carbonyl compounds through compensatory puffing. 


\section{INTRODUCTION}

A large body of evidence suggests that smokers regulate their nicotine intake to maintain a desired and constant blood nicotine level thereby optimising their levels of cognitive arousal, mood and performance. ${ }^{1}$ Considerable data lends support to this theory of self-titration (also known as compensatory smoking or self-regulation), suggesting that smokers adjust their puffing behaviours when given 'light' (low nicotine low tar yield) cigarettes. ${ }^{2-7}$ By increasing their puffing frequency, smokers can extract a greater amount of nicotine from light cigarettes compared to machine-yields. This, however, can increase exposure to carbon monoxide and tar (known to contain many carcinogens). ${ }^{6,8}$ Thus low yield nicotine cigarettes may not necessarily promote harm reduction. ${ }^{9}$ This assertion is further supported by recent data in which reduced nicotine content tobacco cigarettes did not lead to a reduction in expired carbon monoxide and urine 4-(Methylnitrosamino)-1-(3-pyridyl)-1-butanol (NNAL) (major metabolite of the tobaccospecific lung carcinogen (NNK), despite the reported decrease in cigarettes per day; ${ }^{10}$ implying that compensatory puffing may have occurred. Such compensatory puffing behaviour has recently been demonstrated in experienced vapers. In a study using a standardised 10-puff protocol, experienced e-cigarette users increased their puff duration following the use of 0 $\mathrm{mg} / \mathrm{mL}$ compared with $36 \mathrm{mg} / \mathrm{mL}$ nicotine concentration (but not with 8 or $18 \mathrm{vs} 36 \mathrm{mg} / \mathrm{mL})^{11}$. However, the fixed 10-puff protocol may have limited users' ability to engage in compensatory puffing. More recently, ${ }^{12}$ in two separate sessions, Dawkins et al. (2016) asked twelve experienced vapers to use a 'Joyetech eVic' tank-style $(8.5 \mathrm{~W})$ e-cigarette ad libitum in the lab for a period of 60 minutes. Participants were given high $(24 \mathrm{mg} / \mathrm{mL})$ and low $(6 \mathrm{mg} / \mathrm{mL})$ nicotine concentration liquids on two separate days in order to observe changes in puffing topography. Although plasma nicotine concentrations were significantly lower in the $6 \mathrm{mg} / \mathrm{mL}$ condition, 
puff frequency and duration were longer, and the volume of liquid consumed almost doubled. This study, however, did not examine the effects of compensatory puffing on carbonyl levels in the aerosol.

Toxic substances and carcinogens reported in e-cigarette aerosols are at trace or very low levels in comparison to tobacco smoke. ${ }^{13-15}$ However, more intensive puffing patterns combined with higher voltage devices can lead to over-heating the atomiser coil ${ }^{16}$ resulting in increased production of carbonyls such as formaldehyde, acetaldehyde, acetone and acrolein ${ }^{17,18}$ which are all listed by the FDA (U.S. Food and Drug Administration) as harmful or potentially harmful constituents (HPHC) in tobacco products and tobacco smoke. ${ }^{19}$ Formaldehyde is a known human carcinogen and acetaldehyde is classified as possibly carcinogenic to humans (International Agency for Research of Cancer, IARC).${ }^{19}$ Acrolein and acetone are both classified as respiratory irritants and acrolein as a cardiovascular toxicant (FDA). ${ }^{19}$ Whether a more intensive puffing regime associated with using lower nicotine concentration liquid (as demonstrated by Dawkins et al., 2016), increases exposure to carbonyl compounds via aerosols, has not been explored.

This research is particularly timely due to the newly implemented (20th May 2016) European Tobacco Product Directive 2014/40/EU (EU-TPD), under which article 20 imposes a limit of $20 \mathrm{mg} / \mathrm{mL}$ on supply of all nicotine concentration e-cigarette products. By implication, vapers who require higher nicotine concentration will be compelled to switch to a lower nicotine concentration.

This study was designed to assess whether more intensive puffing regimens associated with compensatory behaviours produce higher levels of carbonyls in the e-cigarette aerosols. Human puffing patterns obtained in the Dawkins et al. study ${ }^{12}$ were mimicked using a smoking 
machine and the aerosol composition was analysed. We hypothesised that at the higher nicotine concentration of $24 \mathrm{mg} / \mathrm{mL}$, lower levels of carbonyl compounds will be produced due to the smaller volume of liquid consumed, in comparison to higher carbonyl levels associated with the more intensive puffing topography obtained with the $6 \mathrm{mg} / \mathrm{mL}$ nicotine liquid. 


\section{METHODS}

\section{Human puffing topography data}

The human puffing topography data was taken from Dawkins et al. (2016). In two separate sessions, 12 experienced vapers were asked to use a 'Joyetech eVic Supreme' e-cigarette (output voltage fixed at 3.9 V equipped with an "Aspire Nautilus" tank set to the largest airflow housing a $\mathrm{BVC}$ atomiser with a resistance of $1.8 \mathrm{Ohm}$ resulting in a power of $8.5 \mathrm{~W}$ ) ad libitum for 60 min. All participants were daily e-cigarette users and had used for more than 3 months, were currently using a tank-style device, familiar with $24 \mathrm{mg} / \mathrm{mL}$ nicotine concentration liquid (i.e. used $24 \mathrm{mg} / \mathrm{mL}$ at least once in the last 6 months), used a mean of $11 \mathrm{mg}$ e-liquid per day and had a baseline salivary cotinine level $>100 \mathrm{ng} / \mathrm{mL}$. Participants were $12 \mathrm{~h}$ nicotine abstinent (as confirmed by blood nicotine levels measured at the start of the session). Using a double-blind counterbalanced design, participants were administered a high $(24 \mathrm{mg} / \mathrm{mL})$ and low $(6 \mathrm{mg} / \mathrm{mL})$ nicotine concentration on two separate days. Puffing topography (puff number and puff duration) was recorded by the eVic ${ }^{\mathrm{TM}}$ and downloaded to 'My Vapors Joyetech 1.4' (See Dawkins et al, 2016 for the full protocol).

\section{E-cigarette}

The 'Joyetech eVic Supreme' was fitted with an Aspire Nautilus tank. The device was set up with the same parameters as the Dawkins and colleagues study (see above). Lithium-ion batteries (nominal capacity $2500 \mathrm{mAh}$ ) were charged for $24 \mathrm{~h}$ before each test and replaced when the devices indicated a decrease in charging level from 100 to $20 \%$. Only fully charged batteries were used at the start of each test. The tank was filled with $3.5 \mathrm{~mL}$ nicotine e-liquid $24 \mathrm{hr}$ before the experiment and refilled with $2.5 \mathrm{~mL}$ when levels dropped to $1 \mathrm{~mL}$. 


\section{Materials}

\section{E-liquids}

For both the participants and for the smoking machine, nicotine liquids were selected by firstly identifying the ten most popular brands of nicotine liquid (search conducted of online retailers in January/ February 2015) which were available in nicotine concentrations of 6 and $24 \mathrm{mg} / \mathrm{mL}$ and in tobacco flavour. One member of the research team selected one brand ('ROK Universal') at random with 6 and $24 \mathrm{mg}$ nicotine/mL (both Britannia blend tobacco flavour and the carrier vehicle comprising $>60 \%$ propylene glycol (PG) as stated on the label). To determine whether the carbonyl levels in the carrier vehicle were similar for both concentration products prior to aerosol production, analyses were performed on both 6 and $24 \mathrm{mg} / \mathrm{mL}$ nicotine solutions.

\section{Aerosols generation}

Aerosols were generated using the automatic smoking machine Palaczbot (University of Technology, Lodz, Poland) as described previously. ${ }^{20}$ The e-cigarette was set to the smoking machine at an angle of 45 degrees due to the bottom coil.

The smoking machine was programmed to mirror the puffing topography observed in the Dawkins and colleagues' experiment (see Table 1). Note there was a slight discrepancy in the overall mean puffing topography due to one participant being removed from the final analysis in the Dawkins' et al paper (due to problems obtaining blood samples) in which the human sample is reported as $\mathrm{N}=11$. In the current study all analyses were performed to include the original sample $\mathrm{N}=12$ as all 12 provided complete puffing topography information. 
Taking into account the intensive puffing protocols used in this study, the number of puffs taken for one sampling tube was reduced from the standard 15-puff protocol used in our laboratory to 14 puffs. This procedure was applied to avoid overloading the sampling tube after a series of puffing (the tube was validated for a maximum of $200 \mathrm{mg}$ of aerosol generated per one sample). This protocol was required as the mean amount of aerosol vaporized by the user with $6 \mathrm{mg} / \mathrm{mL}$ was $1.06 \mathrm{~g}$ (200 mg/14 puffs).

Inter-puff intervals (IPIs) were taken from the human puffing topography data in each condition. A second sorbent tube was serially connected to ensure all carbonyl compounds were trapped by the first tube. The e-cigarette was activated by the smoking machine exactly when the puff started and the aerosol was released immediately after the puff was completed. Aerosols from each e-liquid were tested five times and a different tank was used for each e-liquid.

\section{Carbonyl compounds analysis}

The method recommended by the U.S. Environment Protection Agency (EPA) (2003) was applied for the determination of carbonyl compounds as described earlier. ${ }^{18}$ The most commonly reported carbonyl compounds were selected: formaldehyde, acetaldehyde, acetone and acrolein. ${ }^{13,15,16,18}$ The limits of quantification are as follows (per $50 \mu 1$ and 14 puffs), formaldehyde, acrolein and acetone 20 and $60 \mathrm{ng}$ and for acetaldehyde 10 and $30 \mathrm{ng}$.

\section{Statistical analysis}

Statistical calculations were performed using Statistica 12.0 Software (Statsoft, Inc, US). T-tests were performed to explore differences between mean carbonyl levels in aerosols generated from 
the 6 and $24 \mathrm{mg} / \mathrm{mL}$ puffing conditions. For aerosol yield means, since t-test assumptions were not met, U-Mann-Whitney tests were performed.

\section{RESULTS}

\section{Carbonyl compounds in liquids}

Results for carbonyls in liquids are presented in $\mathrm{ng} / 50 \mu \mathrm{L}$. Acrolein was not detected. Formaldehyde was not detected in the $6 \mathrm{mg} / \mathrm{mL}$ e-liquid and was below level of quantification (BLQ) in the $24 \mathrm{mg} / \mathrm{mL}$ e-liquids. Acetaldehyde was detected in ranges of $60-80$ and 40-70 $\mathrm{ng} / 50 \mu \mathrm{L}$ in 6 and $24 \mathrm{mg} / \mathrm{mL}$ e-liquids respectively. Acetone was below the limit of quantification in $6 \mathrm{mg} / \mathrm{mL}$ e-liquids and in range of BLQ - $90 \mathrm{ng} / 50 \mu \mathrm{L}$ in $24 \mathrm{mg} / \mathrm{mL}$ e-liquids.

\section{Carbonyl compounds in aerosols}

Results are presented in mg and ng per one puff (that is, levels of carbonyls divided by 14) and also multiplied by the mean number of puffs taken by users to represent the one hour of ecigarette use as per the human topography data: 74 and 47 puffs in the 6 and $24 \mathrm{mg} / \mathrm{mL}$ nicotine e-liquid conditions respectively. Table 2 shows the amount of each analysed carbonyl compound and e-liquid vaporised in one puff from 6 and $24 \mathrm{mg} / \mathrm{mL}$ nicotine e-liquids. Acrolein was not detected. Formaldehyde, acetaldehyde and acetone were found in all tested aerosols.

The puffing regimen associated with the $6 \mathrm{mg} / \mathrm{mL}$ e-liquid resulted in a $52 \%$ increase in aerosol per puff and $45 \%$ more formaldehyde, $33 \%$ more acetaldehyde and $65 \%$ more acetone. These differences were statistically significant: for the aerosol yield $p=0.005$ (U Mann-Whitney test), formaldehyde $\mathrm{p}=0.03$, acetaldehyde $\mathrm{p}=0.01$ and acetone $\mathrm{p}=0.04$ (t-tests). 
When the results were multiplied by the number of puffs taken by participants per hour (74 and 47 puffs per hour for the 6 and $24 \mathrm{mg} / \mathrm{mL}$ e-liquids respectively) the differences in values further increased (see Table 2; all p values $<0.01$ ).

\section{DISCUSSION}

This study is the first to explore whether a more intensive puffing regimen associated with using a low nicotine concentration liquid $(6 \mathrm{mg} / \mathrm{mL})$ led to increased carbonyl formation compared with a puffing regimen associated with a high nicotine concentration liquid (24 $\mathrm{mg} / \mathrm{mL}$ ). In line with previous studies which found increased levels of toxicants from 'low tar low nicotine yield' compared to 'regular' cigarettes in tobacco smoke, ${ }^{6}$ the current study suggests that compensatory puffing by vapers may increase exposure to carbonyl compounds. Levels of formaldehyde, acetaldehyde and acetone measured in aerosols were greater with puffing regimens associated with the $6 \mathrm{mg} / \mathrm{mL}$ nicotine liquid compared with the $24 \mathrm{mg} / \mathrm{mL}$ nicotine liquid. Consistent with a previous report, ${ }^{18}$ acrolein was not detected in either nicotine liquid aerosols including in the more intensive puffing regimen condition. This is also in agreement with recent findings where acrolein could only be detected when output battery voltage was increased to $20 \mathrm{~W}^{16}$ Due to its long term adverse health effects ${ }^{21,22}$ and reactivity when in contact with glycerine, the presence of acrolein in e-liquid has been a concern. Our data confirms that even under the compensatory puffing regimen at $6 \mathrm{mg} / \mathrm{mL}$, acrolein is not produced.

Similar to previous studies, ${ }^{23}$ levels of carbonyls in both nicotine concentration liquids were minimal compared to levels found in generated aerosols. Indeed, many chemicals are not 
present in the original nicotine liquids but are produced as by-products of the liquid oxidation when converting to aerosol form. ${ }^{24}$ Notably, previous studies found propylene glycol-based liquids have a greater propensity to increase levels of carbonyls formation due to its higher susceptibility to thermal degradation. ${ }^{25}$ Similarly, Bekki and colleagues report potential risks of formaldehyde, acetaldehyde and acrolein formation when propylene glycol and glycerol respectively, come in contact with a heated nichrome coil. ${ }^{24,26}$ Puffing topography and nicotine concentration have been identified as clear influencers of e-cigarette emission particles, ${ }^{27}$ and a recent laboratory study found that puff duration and inter-puff intervals (hereafter referred to as IPI) were key factors influencing aerosol yields. ${ }^{28}$ Our study also provides clear evidence that puffing topography influences e-cigarette aerosol yields. Here, levels of the aerosol yields per one puff were $52 \%$ higher with the puffing regimen associated with the 6 compared with the 24 $\mathrm{mg} / \mathrm{mL}$ condition. Relatedly, the $6 \mathrm{mg} / \mathrm{mL}$ e-liquid condition resulted in $45 \%$ more formaldehyde, $33 \%$ more acetaldehyde and $65 \%$ more acetone. These differences further increased when total number of puffs were taken into account. Thus, longer puff duration and shorter IPIs associated with the use of the $6 \mathrm{mg} / \mathrm{mL}$ nicotine concentration e-liquid contribute to an increase in aerosol yield and higher levels of formaldehyde, acetaldehyde and acetone in aerosol. Nevertheless, whether the increased generation of carbonyls was a sole consequence of increased dosing or if a higher temperature associated with longer puff durations also contributed is unclear. Ascertaining the interacting determinants of increased carbonyl exposure is clearly a priority for further research.

Our findings reinforce the notion that realistic puffing protocols (puffing duration and frequency) used to generate e-cigarette aerosols in smoking machines are key parameters in detecting the presence of carbonyls. However, comparing levels of carbonyls reported previously 
is problematic. The wide variability of carbonyl levels found in e-cigarette aerosols across previous studies may be partly due to: i) the use of differing puffing protocols, ii) different ecigarette devices, iii) in some cases different trapping techniques, iv) different specification smoking machines. For instance, whilst some employed a series of 15 puffs per session with a puffing protocol of $1.8 \mathrm{~s}$ puff duration and IPIs ranging from $10-17 \mathrm{~s}$ to analyse first and second generation devices ${ }^{13,18}$ others used 10 puffs per session, a series of $2 \mathrm{~s}^{24}$ or $3 \mathrm{~s}^{16}$ puff duration with IPIs of 30s to analyse first and third generation devices respectively. Herrington and Myers ${ }^{23}$ configured their smoking machine with series of 10 puffs per session, with longer puff duration of $4 \mathrm{~s}$ and shorter IPIs of $10 \mathrm{~s}$ to analyse first generation e-cigarettes. The use of uniform puffing protocols presents further problems as previous studies show that vapers' puffing patterns differ widely across types of e-cigarettes. ${ }^{29}$ Indeed, such uniform puffing protocols fail to account for the wide variability of use across e-cigarette devices and compensatory puffing patterns exerted with different nicotine concentrations and in turn, do not reflect realistic use or true puffing topography. A key strength of the current study was the use of puffing regimens collected from a sample of human participants rather than hypothetical puffing scenarios. Given that puffing topography (puff duration and frequency) is a key determinant of nicotine delivery and correlates highly with blood nicotine absorption, ${ }^{12}$ adopting the puffing parameters employed in the current study would strengthen the validity and generalizability of future studies investigating potential exposure of carbonyls in e-cigarette aerosols.

Although levels of carbonyls were greater in aerosols from the low nicotine concentration, these levels were still much lower than those reported in tobacco smoke even when comparing one hour of e-cigarette use with one cigarette smoked. Using the ISO Standard 3402 regimen (1999, cited in Counts, Morton, Laffoon, Cox, \& Lipowicz, 2005), previous 
studies found levels between 2 and $50 \mu \mathrm{g}, 30$ and $650 \mu \mathrm{g}$, and 2.5 to $60 \mu \mathrm{g}$ generated per one cigarette in formaldehyde, acetaldehyde and acrolein respectively. ${ }^{30}$

The current study must be interpreted in light of the following limitations. The eVic does not capture puff flow rate and puff volume, consequently these variables could not be incorporated into the smoking machine settings. Nevertheless, studies found that puffing volume ${ }^{28}$ and puff velocity alone do not affect e-liquid evaporation. ${ }^{31}$ Secondly, our analysis is confined to the four major selected carbonyls commonly reported in the literature, ${ }^{13,15,16,18}$ thus our findings must not be confounded with other carbonyls or toxicants possible in e-cigarette aerosols. Thirdly, previous studies found that PG/VG (propylene glycol to vegetable glycerine) ratio can influence carbonyls formation in smoking machines, ${ }^{18}$ and may alter puffing topographies. Here we kept this constant at $>60 \%$ PG vehicle for both nicotine solutions however, the e-liquids were not tested to verify PG/VG ratio or nicotine content. Fourthly, here we aimed to replicate human puffing topography observed in our earlier study, using the same ecigarette device and tobacco flavour nicotine liquid, with group averages for puff duration and IPI. Other flavours ${ }^{32}$, device types and settings may influence the levels of carbonyls in the aerosol and indeed, may be associated with different puffing topographies within and between individuals. $^{29}$ Lastly, controlled clinical experiments may not reflect real-world puffing behaviour, ${ }^{33}$ future studies would therefore benefit from using 'real life' puffing patterns and individual data. Although fixed device settings allow a high degree of experimental control, this may not reflect later generation e-cigarette use outside of the clinic. Notably given the rise in the uptake of subohming (the use of newer generation devices mounted with atomisers of less than 1 $\mathrm{Ohm})$, users commonly reduce their e-liquid nicotine concentration whilst increasing wattage ${ }^{34}$ which may in turn influence users exposure to carbonyls and other HPHS. Overall, although in 
need of replication in a larger sample outside of a clinic setting, this is the first study to provide data on the potential risks of compensatory puffing behaviours in vapers based on actual, rather than hypothetical, puffing patterns.

Using real puffing topography data from a sample of experienced vapers, this study is, to our knowledge, the first to provide empirical evidence that more intensive puffing regimens, as observed in vapers using a lower nicotine strength liquid, may lead to an increase in exposure to inhaled carbonyl compounds. The cap on nicotine concentration at $20 \mathrm{mg} / \mathrm{mL}$ set by the EU-TPD may therefore have the unintended consequence of encouraging use of lower nicotine containing e-liquid which in turn, may increase exposure to carbonyl compounds through compensatory puffing. Although in need of replication outside of a laboratory setting and with a wider range of nicotine concentration e-liquids, this study suggests that future regulatory decision makers should carefully consider where to set the upper limit on nicotine e-liquid concentrations.

Table 1. Puffing regimens

\begin{tabular}{|c|c|c|c|c|}
\hline Nicotine & Puff duration & Inter-puff & Number of & Amount of liquid \\
\hline centration & & Interval & puffs & consumed $[\mathrm{g}]$ \\
\hline
\end{tabular}

$\begin{array}{lllcc}6 \mathrm{mg} / \mathrm{mL} & 5.04 \mathrm{~s} & 44.3 \mathrm{~s} & 74 & 1.06 \\ 24 \mathrm{mg} / \mathrm{mL} & 3.76 \mathrm{~s} & 74.5 \mathrm{~s} & 47 & 0.344\end{array}$


Table 2. Levels of aerosol and carbonyl compound yields generated from 6 and $24 \mathrm{mg} / \mathrm{mL}$ nicotine e-liquids, per one puff and, for all puffs as per human topography data

\begin{tabular}{|c|c|c|c|}
\hline \multicolumn{2}{|c|}{ Arocol viold and colocted corbonol } & \multicolumn{2}{|r|}{$24 \mathrm{mg} / \mathrm{mL}$ (3.76s puff) } \\
\hline \multicolumn{2}{|c|}{ Aerosol yield and selected carbonyl } & $\begin{array}{l}\text { Level per puff [ng unless } \\
\text { otherwise specified] }\end{array}$ & $\begin{array}{l}\text { Level per puff [ng unless } \\
\text { otherwise specified] }\end{array}$ \\
\hline \multirow[t]{2}{*}{ yield $[\mathrm{mg}]$} & Mean \pm SD & $11.1 \pm 1.8$ & $7.3 \pm 0.5$ \\
\hline & Median (Q1-Q3) & $11.3(9.1-12.9)$ & $7.3(6.9-7.7)$ \\
\hline \multirow[t]{2}{*}{ Formaldehyde } & Mean \pm SD & $46.1 \pm 12.8$ & $31.7 \pm 6.4$ \\
\hline & Median (Q1-Q3) & $44.8(35.0-52.4)$ & $33.9(28.8-35.7)$ \\
\hline \multirow[t]{2}{*}{ Acetaldehyde } & Mean \pm SD & $29.3 \pm 4.9$ & $22.1 \pm 2.7$ \\
\hline & Median (Q1-Q3) & $29.5(24.3-31.6)$ & $22.2(20.8-24.3)$ \\
\hline \multirow[t]{2}{*}{ Acetone } & Mean \pm SD & $9.9 \pm 2.7$ & $6.0 \pm 3.1$ \\
\hline & Median (Q1-Q3) & $10.5(7.9-11.6)$ & $5.1(4.0-6.9)$ \\
\hline Acrolein* & Mean \pm SD & ND & ND \\
\hline \multicolumn{2}{|c|}{$\begin{array}{l}\text { Aerosol yield and selected carbonyl } \\
\text { compounds for all puffs }\end{array}$} & $\begin{array}{l}\text { Level for all } \\
\text { (74) puffs [ [ } \mathrm{g} \text { unless } \\
\text { otherwise specified }]\end{array}$ & $\begin{array}{l}\text { Level for all } \\
\text { (47) puffs [ } \mu \mathrm{g} \text { unless } \\
\text { otherwise specified] }\end{array}$ \\
\hline \multirow[t]{2}{*}{ yield $[\mathrm{mg}]$} & Mean \pm SD & $822 \pm 137$ & $342 \pm 22$ \\
\hline & Median (Q1-Q3) & $835(671-956)$ & $342(326-363)$ \\
\hline \multirow[t]{2}{*}{ Formaldehyde } & Mean \pm SD & $3.41 \pm 0.94$ & $1.49 \pm 0.30$ \\
\hline & Median (Q1-Q3) & $3.31(2.59-3.88)$ & $1.59(1.35-1.68)$ \\
\hline \multirow[t]{2}{*}{ Acetaldehyde } & Mean \pm SD & $2.17 \pm 0.36$ & $1.04 \pm 0.13$ \\
\hline & Median (Q1-Q3) & $2.19(1.80-2.34)$ & $1.05(0.98-1.14)$ \\
\hline \multirow[t]{2}{*}{ Acetone } & Mean \pm SD & $0.73 \pm 0.20$ & $0.28 \pm 0.14$ \\
\hline & Median (Q1-Q3) & $0.78(0.58-0.86)$ & $0.24(0.19-0.32)$ \\
\hline Acrolein* & Mean \pm SD & ND & ND \\
\hline
\end{tabular}

Note: $\mathrm{ng}=$ nanogram; $\mathrm{mg}=$ milligram; $\mu \mathrm{g}=$ microgram; $\mathrm{ND}=$ not detected; All $\mathrm{p}$ values for aerosol yield and carbonyl compounds per puff were $<0.05$. All $p$ values for aerosol yield and carbonyl compounds for all puffs were $<0.01$;

* statistical comparison between condition not conducted as carbonyl was ND. 
Funding This work was supported by a fully-funded University of East London PhD Excellence Scholarship to CK.

Declaration of interests We have read and understood the journal's policy on declaration of interests and declare the following. We have received no support from any companies for this work and no non-financial conflicts of interest that would be considered relevant to this work. All authors have completed the Unified Competing Interest form at www.icmje.org/coi_disclosure.pdf (available on request from the corresponding author). LK worked in the Institute of Occupational Medicine and Environmental Health, which is a public body, which, besides research works, offers expertise and conduct analyses on behalf of the Polish government and the industry. LK works as an expert for the Polish National Committee for Standardization and for the European Committee for standardization of requirements and test methods for e-liquids and emissions.

CFK and OC declare no competing interests.

JK works in the Institute of Occupational Medicine and Environmental Health, which is a public body, which, besides research, offers expertise and conduct analyses on behalf of the Polish government and the industry.

LED has previously (2010-2013) conducted research for several independent electronic cigarette companies. These companies had no input into the design, conduct or write up of the projects. She has also acted as a consultant for the pharmaceutical industry and as an expert witness in a patent infringement case (2015). 
Contributors LED and LK conceptualised the project. LK and JK conducted the chemical analysis. LK, CFK, OC and LED handled the interpretation of the data. CFK handled the drafting of the manuscript with substantial input from LED, LK and OC. All the named authors contributed substantially to the writing of the manuscript.

We further confirm that this work has not been published and is not considered in any other journals for publication.

Data sharing Additional unpublished data and information are available to the editorial and reviewers team upon request.

Permission Not applicable, although the human topography data derived from a related study published by the same group (Dawkins, Kimber, Doig, Feyerabend \& Corcoran, 2016). 


\section{REFERENCES}

1. Benowitz NL. Compensatory smoking of low-yield cigarettes. Natl Cancer Institute Risks Assoc with Smok cigarettes with low Mach yields tar nicotine Smok Tob Control Monogr No. 2001;13:39-63.

2. Ashton H, Stepney R, Thompson JW, Rawbone RG. Self-titration by cigarette smokers. Br Med J. 1979;2(6192):731-732. http://www.scopus.com/inward/record.url?eid=2-s2.00018798575\&partnerID=40\&md5=30a018ac8dc2ec73d13e404f9d49c4e0.

3. Ashton H, Watson DW, Marsh R, Sadler J. Puffing frequency and nicotine intake in cigarette smokers. Br Med J. 1970;3(5724):679-681. doi:10.1136/bmj.3.5724.679.

4. Herning RI, Jones RT, Bachman J, Mines AH. Puff volume increases when low-nicotine cigarettes are smoked. Br Med J (Clinical Res Ed. 1981;283(6285):187-189.

http://search.ebscohost.com/login.aspx?direct=true \&db=a9h\&AN=4949103\&site=ehostlive.

5. Scherer G. Smoking behaviour and compensation: a review of the literature. Psychopharmacol. 1999;145(1):1-20.

http://search.ebscohost.com/login.aspx?direct=true \&db=a9h\&AN=4694245\&site=ehostlive.

6. Strasser AA, Lerman C, Sanborn PM, Pickworth WB, Feldman EA. New lower nicotine cigarettes can produce compensatory smoking and increased carbon monoxide exposure. Drug Alcohol Depend. 2007;86(2/3):294-300. doi:10.1016/j.drugalcdep.2006.06.017.

7. Sutton SR, Russell MAH, Iyer R, Feyerabend C, Saloojee Y. Relationship between cigarette yields, puffing patterns, and smoke intake: evidence for tar compensation? $\mathrm{Br}$ Med J (Clinical Res Ed. 1982;285(6342):600-603. 
http://search.ebscohost.com/login.aspx?direct=true \&db=a9h\&AN=4937613\&site=ehostlive.

8. Hecht SS, Murphy SE, Carmella SG, et al. Effects of reduced cigarette smoking on the uptake of a tobacco-specific lung carcinogen. J Natl Cancer Inst. 2004;96(2):107-115.

9. Ebert R V, McNabb ME, McCusker KT, Snow SL. Amount of nicotine and carbon monoxide inhaled by smokers of low-tar, low-nicotine cigarettes. JAMA.

1983;250(20):2840-2842. doi:10.1001/jama.1983.03340200074034.

10. Donny EC, Denlinger RL, Tidey JW, et al. Randomized Trial of Reduced-Nicotine Standards for Cigarettes. N Engl J Med. 2015;373(14):1340-1349. doi:10.1056/NEJMsa1502403.

11. Ramôa CP, Hiler MM, Spindle TR, et al. Electronic cigarette nicotine delivery can exceed that of combustible cigarettes: a preliminary report. Tob Control. 2015. doi:10.1136/tobaccocontrol-2015-052447.

12. Dawkins LE, Kimber CF, Doig M, Feyerabend C, Corcoran O. Self-titration by experienced e-cigarette users: blood nicotine delivery and subjective effects. Psychopharmacology (Berl). 2016;233(15-16). doi:10.1007/s00213-016-4338-2.

13. Goniewicz ML, Knysak J, Gawron M, et al. Levels of selected carcinogens and toxicants in vapour from electronic cigarettes. Tob Control. 2014;23(2):133-139. doi:10.1136/tobaccocontrol-2012-050859.

14. Oh AY, Kacker A. Do electronic cigarettes impart a lower potential disease burden than conventional tobacco cigarettes?: Review on e-cigarette vapor versus tobacco smoke. Laryngoscope. 2014;124(12):2702-2706.

http://onlinelibrary.wiley.com/store/10.1002/lary.24750/asset/lary24750.pdf? $v=1 \& \mathrm{t}=\mathrm{irjcj} 8$ 
81\&s=7e07ac95dd0ca8636f735099e2da3ce1db733359.

15. Tayyarah R, Long GA. Comparison of select analytes in aerosol from e-cigarettes with smoke from conventional cigarettes and with ambient air. Regul Toxicol Pharmacol. 2014;70(3):704-710. doi:http://dx.doi.org/10.1016/j.yrtph.2014.10.010.

16. Geiss O, Bianchi I, Barrero-Moreno J. Correlation of volatile carbonyl yields emitted by e-cigarettes with the temperature of the heating coil and the perceived sensorial quality of the generated vapours. Int J Hyg Environ Health. 2016;219(3):268-277. doi:10.1016/j.ijheh.2016.01.004.

17. Farsalinos KE, Voudris V, Poulas K. E-cigarettes generate high levels of aldehydes only in “dry puff” conditions. Addiction. 2015;110(8):1352-1356. doi:10.1111/add.12942.

18. Kosmider L, Sobczak A, Fik M, et al. Carbonyl compounds in electronic cigarette vapors: Effects of nicotine solvent and battery output voltage. Nicotine Tob Res. 2014;16(10):1319-1326. http://search.ebscohost.com/login.aspx?direct=true\&db=psyh\&AN=2014-37086005\&site= $=$ ehost-live.

19. FDA. Harmful and potentially harmful constituents in tobacco products and tobacco smoke; established list. Fed Regist. 2012;77(64):20034-20037. http://scholar.google.com/scholar?hl=en\&btnG=Search\&q=intitle:Harmful+and+Potential ly+Harmful+Constituents + in + Tobacco + Products + and + Tobacco + Smoke + +Established + Li st\#0.

20. Goniewicz ML, Kuma T, Gawron M, Knysak J, Kosmider L. Nicotine Levels in Electronic Cigarettes. Nicotine Tob Res. 2013;15(1):158-166. http://search.ebscohost.com/login.aspx?direct=true\&db=a9h\&AN=84556692\&site=ehost- 
live.

21. DeJarnett N, Conklin DJ, Riggs DW, et al. Acrolein exposure is associated with increased cardiovascular disease risk. $J$ Am Heart Assoc. 2014;3(4). doi:10.1161/JAHA.114.000934.

22. Stevens JF, Maier CS. Acrolein: Sources, metabolism, and biomolecular interactions relevant to human health and disease. Mol Nutr Food Res. 2008;52(1):7-25. doi:10.1002/mnfr.200700412.

23. Herrington JS, Myers C. Electronic cigarette solutions and resultant aerosol profiles. $J$ Chromatogr A. 2015;1418:192-199. doi:10.1016/j.chroma.2015.09.034.

24. Bekki K, Uchiyama S, Ohta K, Inaba Y, Nakagome H, Kunugita N. Carbonyl Compounds Generated from Electronic Cigarettes. Int J Env Res Public Heal. 2014;11(11):1119211200. doi:10.3390/ijerph111111192.

25. Rossiter WJ, Godette M, Brown PW, Galuk KG. An investigation of the degradation of aqueous ethylene glycol and propylene glycol solutions using ion chromatography. Sol Energy Mater. 1985;11(5-6):455-467. doi:10.1016/0165-1633(85)90016-4.

26. Uchiyama S, Ohta K, Inaba Y, Kunugita N. Determination of Carbonyl Compounds Generated from the E-cigarette Using Coupled Silica Cartridges Impregnated with Hydroquinone and 2,4-Dinitrophenylhydrazine, Followed by High-Performance Liquid Chromatography. Anal Sci. 2013;29(12):1219-1222. doi:10.2116/analsci.29.1219.

27. Fuoco FCC, Buonanno G, Stabile L, Vigo P. Influential parameters on particle concentration and size distribution in the mainstream of e-cigarettes. Environ Pollut. 2014;184:523-529. doi:10.1016/j.envpol.2013.10.010.

28. Kosmider L, Madej D, Garwon M, Sobczak A. Influence of Electronic Cigarettes Puffing Regimes on Amount of Vaporized Liquid. Przeglqd Lek. 2016;73(10):699-703. 
29. Behar RZ, Hua M, Talbot P. Puffing topography and nicotine intake of electronic cigarette users. PLoS One. 2015;10(2):e0117222. doi:10.1371/journal.pone.0117222.

30. Counts ME, Morton MJ, Laffoon SW, Cox RH, Lipowicz PJ. Smoke composition and predicting relationships for international commercial cigarettes smoked with three machine-smoking conditions. Regul Toxicol Pharmacol. 2005;41(3):185-227. doi:10.1016/j.yrtph.2004.12.002.

31. Talih S, Balhas Z, Eissenberg T, et al. Effects of User Puff Topography, Device Voltage, and Liquid Nicotine Concentration on Electronic Cigarette Nicotine Yield: Measurements and Model Predictions. Nicotine Tob Res. 2014. doi:10.1093/ntr/ntu174.

32. Leigh NJ, Lawton RI, Hershberger PA, Goniewicz ML. Flavourings significantly affect inhalation toxicity of aerosol generated from electronic nicotine delivery systems ( ENDS ). BMJ. 2016:1-7. doi:10.1136/tobaccocontrol-2016-053205.

33. Robinson RJ, Hensel EC, Morabito PN, Roundtree KA. Electronic Cigarette Topography in the Natural Environment. PLoS One. 2015;10(6):1-14. doi:10.1371/journal.pone.0129296.

34. Wagener TL, Floyd EL, Stepanov I, et al. Have combustible cigarettes met their match? The nicotine delivery profiles and harmful constituent exposures of second-generation and third-generation electronic cigarette users. Tob Control. 2016. doi:10.1136/tobaccocontrol-2016-053041. 